\title{
Design and Development of a Novel Paper-based Inkjet-Printed RFID-Enabled UHF (433.9 MHz) Sensor Node
}

\author{
Rushi Vyas, Amin Rida, Li Yang and Manos M. Tentzeris \\ School of ECE, Georgia Institute of Technology, Atlanta, GA 30332-0250, USA \\ E-mail: Rushi.Vyas@gatech.edu
}

\begin{abstract}
In this paper, inkjet-printed RFID-enabled sensor modules are investigated for the first time on paper-based substrates for UHF sensing/monitoring applications. An inkjet-printed antenna using silver nano particles on paper substrate is presented and utilized for the compact implementation of the wireless part of the node. The efficiency of the modulation scheme of the sensor signal is characterized at the UHF RFID Band operating around the center frequency of $433.9 \mathrm{MHz}$, verifying the feasibility of the sensor/rfid integration. Such low-cost integrated sensing nodes would enable the deployment of large ad-hoc wireless sensor networks that would mark a revolution in wireless automatic identification and real-time monitoring for medical, inventory and security applications.
\end{abstract}

\section{Introduction}

The growing demand for economic, low power, conformal and durable wireless nodes with sensing capabilities is driven by applications such as: item-level (e.g. storage and handling of food/agricultural products) tracking of temperature and humidity, pharmaceutical logistics, transport and storage of medical products and biosensing among others. The major challenges in today's wireless sensor network applications is the need for lowcost eco-friendly wireless sensor nodes that can be easily implemented out in different environments [1]. RFID is a compact low-power wireless technology regarded as one of the best solutions for the realization of ubiquitous sensor networks [1]. In addition to operating in the unoccupied UHF bands around 433.92 and $910 \mathrm{MHz}$ in the US, RFID tags, at a few cents, cost a fraction of most commonly used wireless sensor nodes. Plus, RFIDs already have an established protocol in UHF (EPC Gen-2) that allow for multi-tag tracking from a single RFID reader station. Furthermore, the inkjet printing on organic substrates, such as paper, makes them inexpensive candidates as packaging and integration platform materials for RFID-enabled wireless sensor nodes in Wireless Sensor Networks (WSN) applications.

\section{Inkjet Printed UHF Antenna on Paper}

One of the main limiting factors of the RFID wireless part is the size of the antenna. For the RFID sensor module introduced in this paper a low cost flexible UHF antenna was printed on paper using silver ink. The dielectric constant $\left(\varepsilon_{\mathrm{r}}\right)$ and loss tangent $(\tan \delta)$ of paper that had been previously determined by the authors was used [2]. With Inkjet-printing, single ink droplet from the nozzle is sprayed to the desired position, thereby minimizing waste of chemicals yielding a cheap and eco-friendly solution.

The design of choice was a half-wavelength taperedwidth U-shaped antenna [2], shown in figure 1. This antenna was printed on paper using silver paste. The tapered width of the two arms offers increased bandwidth and a smaller size for $433.9 \mathrm{MHz}$. The dimensions of this antenna are $8 \mathrm{~cm} \times 15 \mathrm{~cm}$, although more narrowband implementations would allow for much more compact designs, while requiring higher printing accuracies. The Return Loss (RL) plot is shown in figure 2 below. The VSWR of 2 or RL of $-9.6 \mathrm{~dB}$ defines the bandwidth of operation. This antenna operates from $427.14 \mathrm{MHz}$ to $455.27 \mathrm{MHz}$, centred around $440 \mathrm{MHz}$ and matched to a 50 $\mathrm{Ohm}$ load. Since this antenna belongs to the halfwavelength dipole family, it exhibits a quasiomnidirectional radiation pattern.

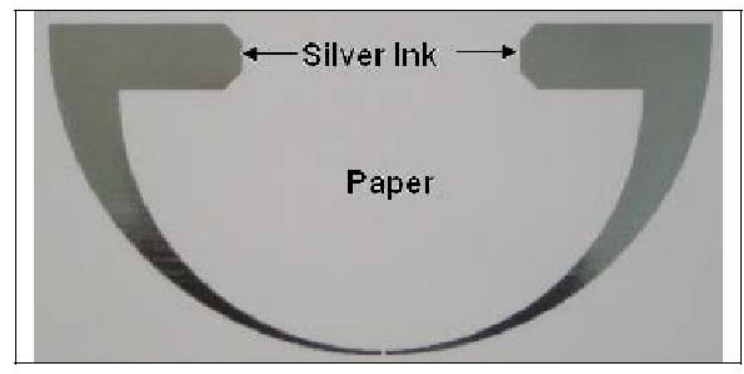

Figure 1. Silver based half-wavelength tapered-width Ushaped antenna 


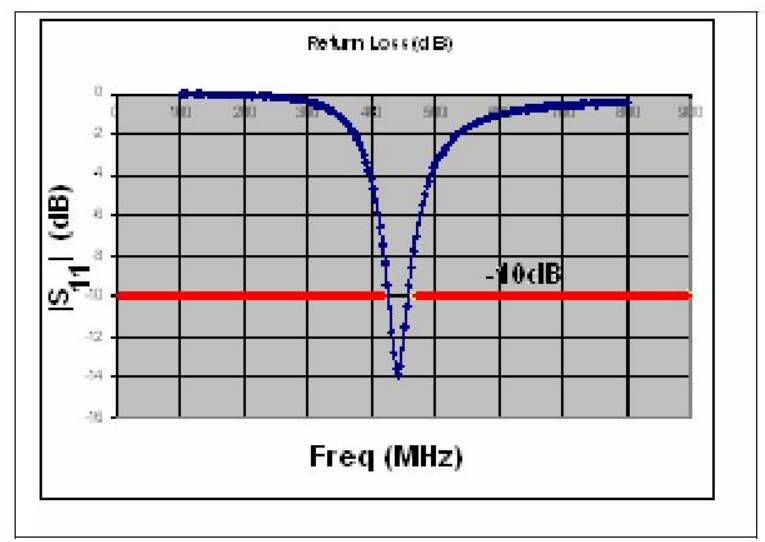

Figure 2. Return loss (dB) of inkjet printed U-shaped antenna on paper.

\section{Sensor and IC Integration}

As a proof of concept, an active "RFID module"-enabled temperature sensor was developed that could function as a low-cost environment-agile node in Wireless Sensor AdHoc Networks. The core of the RFID module comprised of a specially developed IC chip (IC) that combined the limited capabilities of a microcontroller unit (MCU) with a low cost communication front-end module that was configured to interface with sensor and communicate using RFID's UHF Gen-2 protocol at $433.92 \mathrm{MHz}$. For the RFID module prototype, a low cost precision analog output CMOS integrated circuit temperature sensor known as the LM94022 made by National Semiconductor was used. The LM94022 was chosen for its low power consumption (typically $16.2 \mu \mathrm{W}$ ), and its extremely wide temperature range (-50 to 150 degree Celsius) making it versatile for applications ranging from automobiles to a number of electric/electronics appliances and medical applications [3].

\section{A. Circuit Hardware}

The LM94022 was interfaced to a 10 bit analog-to-digital converter, built-in into the IC using a 6 " by 3 "solderable epoxy board with copper plating on one side. The IC was interfaced to the antenna matched at $50 \Omega$ through a $3.5 \mathrm{~mm}$ SMA connector via very short traces soldered onto the epoxy board to minimize losses due to parasitics introduced by the traces [4]. The RFID module comprising of the IC and sensor was powered by a $3 \mathrm{~V}$ Lithium-ion battery made by Panasonic-BSG. For communication (COMM), an external quartz crystal oscillator was tied to the Phase Locked Loop unit (PLL) within the IC to precisely generate a UHF carrier frequency of $433.92 \mathrm{MHz}$. The power efficient operation of the RFID module was enabled by a user triggered push button switch that was connected to the logic controller of the IC. The circuit schematic of the module is shown in Figure 3.

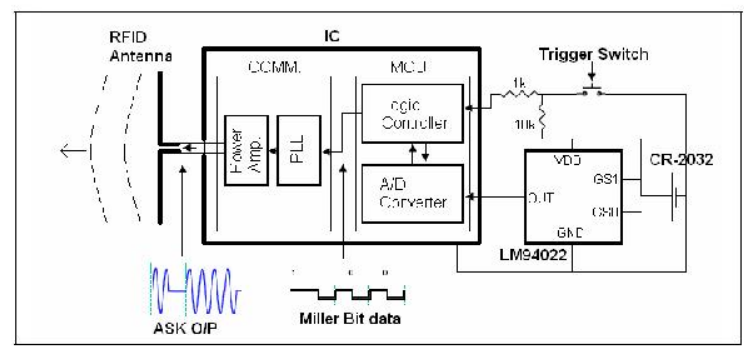

Figure 3. Circuit Schematic of RFID module.

\section{B. ASK Modulation}

In order to verify the integrity and the quality of the data transmission from the RFID module over a maximum range of about 7.5 meters, the IC was initially programmed to generate and send out a regular periodic Amplitude Shift Key (ASK) modulated signal with $50 \%$ duty cycle into the RFID antenna with a carrier frequency of $433.92 \mathrm{MHz}$. On the receiver side, a wideband XR-400 RFID reader antenna, covering the UHF range, was connected to the Tektronix RSA 3408A Real Time Spectrum Analyzer (RTSA) to observe the integrity of the received ASK modulated signal. The initial ASK modulated signals with $50 \%$ duty cycle measured by the RTSA are shown in power vs. frequency and power vs. time scale plots in figures 4 and 5 respectively.

\section{Baud Rate and Bit Encoding}

The Microcontroller unit (MCU) was programmed to ensure that the communication unit (COMM) within the IC communicated at a baudrate in excess of 5kbps as per RFID UHF Gen-2 protocols for miller sub-carrier bit encoding [5]. This was achieved through the use of subroutines in the IC code that produced accurate time delays taking into consideration, the internal timing of the MCU and additional delays that occurred due to the transitions of the program counter within the IC code. For the module prototype, communication was carried out at a baud rate $6.814 \mathrm{kbps}$, which can be calculated by taking the reciprocal of the measured bit duration in figure 5 .

The sensor information was encoded using the 2 subcarrier cycle miller bit encoding shown in figure 6 , which has a lower baud rate requirement as per RFID UHF Gen-2 protocols [5] thereby making it conducive for longer range communication. 


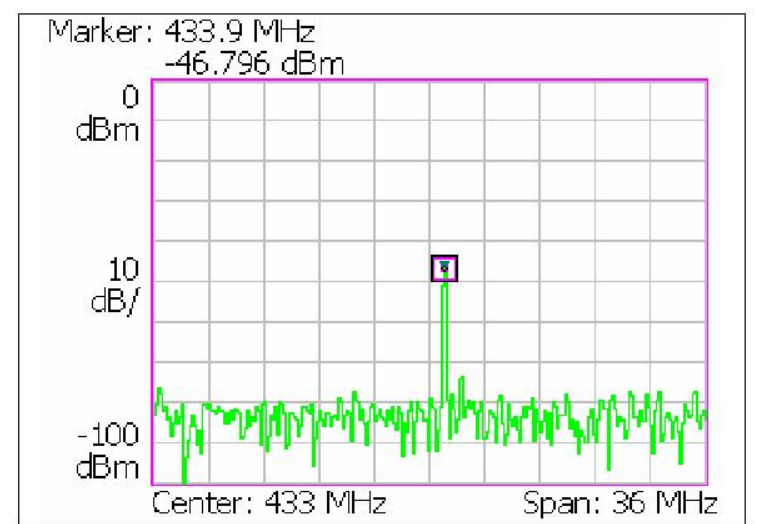

Figure 4. RTSA Received ASK modulated signal with $50 \%$ duty cycle. (Power vs. freq.)

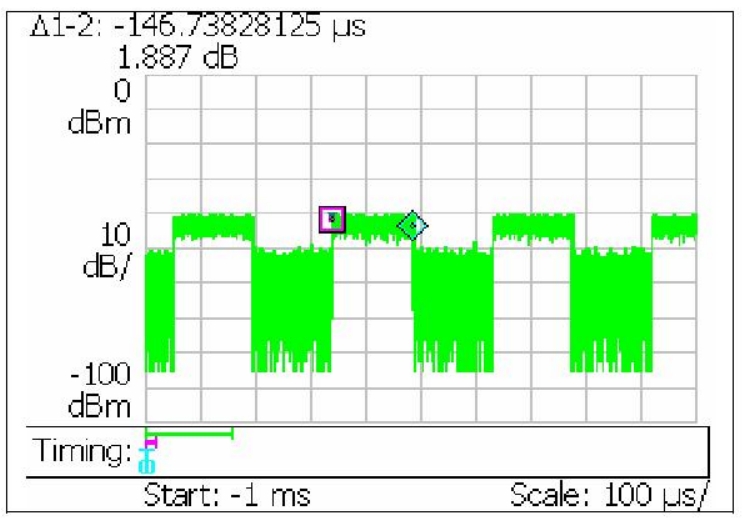

Figure 5. RTSA Measured ASK modulated signal with 50\% duty cycle. (Power vs. Time)

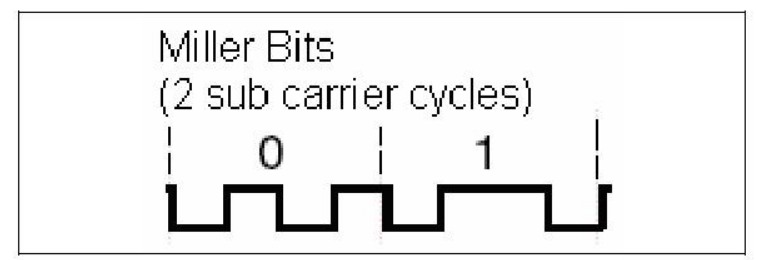

Figure 6. Miller Bit Encoding.

\section{Sensor Operation}

As reported before, the power-efficient operation of the RFID sensor module was initiated through the push of a "push"-button switch, which would wake the IC up from the sleep mode, an extremely low powered state (typically 1.8 $\mu \mathrm{W}$ ) to conserve battery power. Upon wake up (typically $1.5 \mathrm{~mW}$ ), the IC was programmed to sample the analog output of the LM94022 sensor only once in order to conserve battery power, and perform a 10 bit analog to digital conversion of the sensor output. In the idle mode, the CR2032 would be capable of supplying power to the RFID sensor module for approximately 450 hours. The logic controller within the IC was then programmed to encode the converted digital sensor data using RFID UHF Gen-2 miller bit encoding, and place "start" and "stop" flags at the beginning and ending of the encoded data bits. The logic controller modulated the communication unit within the IC in sequence with respect to the encoded sensor data, with the most significant data bit sent out first, which was read using the RTSA on the receiver side. The RFID sensor module was tested at temperatures of 20 and $75^{\circ}$ Celsius, the measurement results of which are shown in figures 7 and 8 respectively on a $10 \mathrm{~ms}$ scale. A comparison of the measured vs. theoretical sensor results is presented in table 1 . Note that the sensor output voltage and its corresponding $\mathrm{A} / \mathrm{D}$ output at $20^{\circ} \mathrm{Celsius}$ is higher than that at $75^{\circ}$ Celsius because the output voltage of the sensor is inversely proportional to the temperature in the region around the sensor [3].

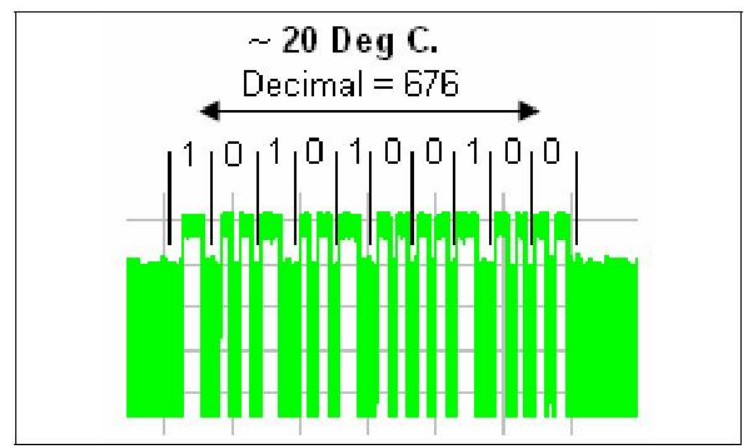

Figure 7. RTSA Measured signal modulation for sensor output at $20^{\circ}$ Celsius. (Power vs. Time)

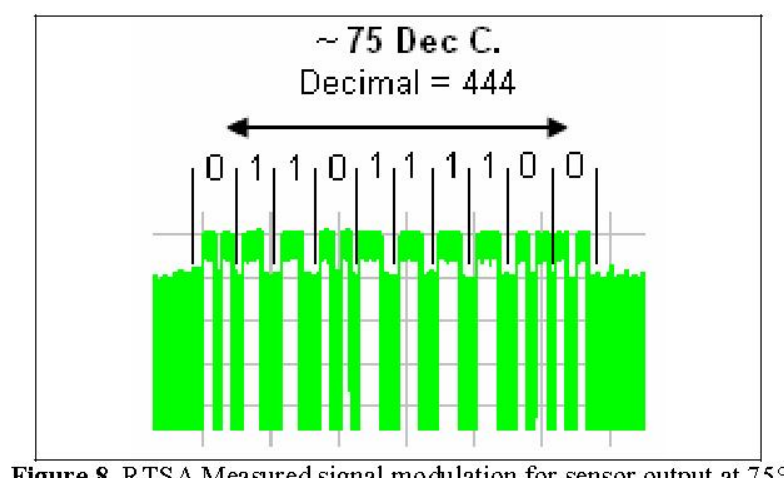

Figure 8. RTSA Measured signal modulation for sensor output at $75^{\circ}$ Celsius. (Power vs. Time) 
Table 1. Actual vs. Measured Results.

\begin{tabular}{|c|c|c|c|c|c|}
\hline $\begin{array}{c}\text { Temp } \\
\left({ }^{\circ} \mathrm{C}\right)\end{array}$ & $\begin{array}{c}\text { Sensor } \\
\text { Output } \\
\text { Actual } \\
(\mathrm{V})\end{array}$ & $\begin{array}{c}\text { Sensor } \\
\text { Output } \\
\text { Meas. } \\
(\mathrm{V})\end{array}$ & $\begin{array}{c}\text { A/D } \\
\text { Output } \\
\text { Actual } \\
(\mathrm{Dec})\end{array}$ & $\begin{array}{c}\text { A/D } \\
\text { Output } \\
\text { Meas. } \\
(\mathrm{Dec})\end{array}$ & $\begin{array}{c}\text { Diff } \\
\left({ }^{\circ} \mathrm{C}\right)\end{array}$ \\
\hline $\mathbf{2 0}$ & 1.899 & 1.909 & 672.21 & 676 & 0.92 \\
\hline $\mathbf{7 5}$ & 1.249 & 1.254 & 442 & 444 & 0.5 \\
\hline
\end{tabular}

- $\quad$ Sensor Output Actual: Sensor Output Measured with multimeter in Volts.

- Sensor Output Meas.: Sensor Output captured by A/D Converter in Volts

- A/D Output Actual: Actual A/D Output at 20 and $75{ }^{\circ} \mathrm{C}$ computed from [3] in decimal format.

- $\quad$ A/D Output Meas: Measured A/D output captured by IC and transmitted to RTSA in decimal format.

The temperatures measured and communicated by the IC were found to be lower by 0.92 and $0.5{ }^{\circ} \mathrm{C}$ for the measurements of the 20 and $75^{\circ} \mathrm{C}$. The discrepancy is most likely due to the limitations of the test set-up since the temperatures were induced in the vicinity of the LM94022 sensor using a heat gun in an open lab environment resulting in unstable temperature conditions, which were measured by the IC. A more accurate setup would require a more stable temperature environment than the one used along with more thorough averaging of the sensor output by the IC to average out minor temperature variations during the short interval of the measurement time. Still these results demonstrate the excellent potential of RFID modules as low-cost low-power platforms of real-time sensing applications.

\section{Conclusion}

A paper-based prototype for a UHF wireless sensor node utilizing active RFID technology was successfully designed for temperature sensing featuring a successful performance.. Various issues concerning hardware, sensing and communication issues were addressed simultaneously for the first time. While for the current design, the IC, sensor and battery were assembled on an epoxy circuit board, and the antenna printed on paper, future wireless prototypes, to be presented at the conference, including packaging will be completely integrated in paper-based substrates. While the current wireless sensor node module was implemented at the UHF band around $433.92 \mathrm{MHz}$ as a proof of concept, future prototypes will be extended to operate at the UHF band around $910 \mathrm{MHz}$.

\section{Acknowledgments}

The authors would like to acknowledge the support of Georgia Electronic Design Center (GEDC) and the NSF CAREER Award.

\section{REFERENCES}

[1] “Sensing New RFID Opportunities" rfid journal,Jan 2006.

[2] A. Rida, L. Yang, M. Tentzeris "Design and Characterization of Novel Paper-based Inkjet-Printed UHF

Antennas for RFID and Sensing Applications" IEEE APS Symposium, pp 2-3, June 2007.

[3] "LM94022: 1.5V, SC70, Multi-Gain Analog Temperature Sensor with Class-AB Output". National Semiconductor Corp., March 2007.

[4] D. M. Pozar, Microwave Engineering, 2nd ed. New York: Wiley, 1998.

[5] "UHF Gen-2 System Overview". Texas Instruments, Sept 2005. 